\title{
Dynamics of Mandelbrot Set with Transcendental Function
}

\author{
Shafali Agarwal \\ Dept. of Computer Applications \\ JSSATE, Noida, India
}

\author{
Gunjan Srivastava \\ Dept. of Computer Applications \\ JSSATE, Noida, India
}

\author{
Dr. Ashish Negi \\ Dept. of Computer Science \\ G. B. Pant Engg. College \\ Pauri Garwal, Uttarakhand, India
}

\begin{abstract}
These days Mandelbrot set with transcendental function is an interesting area for mathematicians. New equations have been created for Mandelbrot set using trigonometric, logarithmic and exponential functions. Earlier, Ishikawa iteration has been applied to these equations and generate new fractals named as Relative Superior Mandelbrot Set with transcendental function.

In this paper, the Mann iteration is being applied on Mandelbrot set with sine function i.e. $\sin \left(z^{n}\right)+c$ and new fractals with the concept of Superior Transcendental Mandelbrot Set will be shown.

Our goal is to focus on the less number of iterations which are required to obtain fixed point of function $\sin \left(z^{n}\right)+c$.
\end{abstract}

Keywords-Superior Transcendental Mandelbrot Set; Mann iteration; fixed point; Escape Criteria.

\section{INTRODUCTION}

Complex dynamics have a new significant development with the explosion of popular interest in the beautiful fractal objects that form the subject matter of the theory $[5,6]$. The computer-generated images of Julia and Mandelbrot sets bombard mathematicians to investigate the nature of both the Fatou and Julia sets of a given complex function [4].

Though a moment's reflection confirms the origin is a critical point for the cosine and for the sine, the transcendental Julia sets fill major regions of the complex plane [3].

Based on this concept, authors found the region $M$ of nonescape corresponds to a principal central bulb set with a fractal series of black hearts, including a series lining the $x$-axis, and that any $c$ value in $M$ corresponds precisely to Julia set kernels of the corresponding quadratic type [7].

Ereneko [1] studied that for every transcendental functions, the set of escaping points is always non-empty. The set of parameter values of $c$ for which the Julia set of $Q$ is connected forms the well-known Mandelbrot set.

Mandelbrot set serves as a lexicon for the Julia Set. The location of the parameter $c$ with in the Mandelbrot set furnishes information on properties of the corresponding Julia set. There are similarities between magnified positions of the Mandelbrot set and the corresponding filled Julia set holds only near certain $c$ values such as the central junctions of the antenna. These are $c$ values, for which 0 is eventually periodic; such $c$ values are called Misiurcwicz points [10]. In
2004, Rani jointly with Kumar applied Mann iteration to functions and introduces superior iteration in non linear sciences and gave new escape criterions for complex polynomials. Thus authors computed superior Julia set [8] and superior Mandelbrot set [9] for complex polynomials.

Fixed point can be obtained by repeated function iteration or Picard iteration. There are some other iteration processes like Ishikawa iteration and Mann iteration, which is required to obtain weak or strong convergence to a fixed point in case of non-expansive maps, pseudocontrative maps etc [6]. The purpose of this paper is to generate superior Julia set and Mandelbrot set using transcendental sine function. We are generating fractals for $\sin \left(z^{n}\right)+c$ using Mann iteration and calculating the fixed points for the same.

\section{DEFINITIONS \& PRELIMINARIES}

The generation of fractal for $\sin \left(z^{n}\right)+c$ is much similar to standard quadratic equation of Mandelbrot set but it consists of repeated iterations upto $n$ times with respect to sine function. Following are some basic definitions require for subsequent analysis.

\section{A. Definition}

(Superior iterates) Let $X$ be a non-empty set of real numbers and $f: X->X$. For $x_{0}$ belongs to $X$, construct a sequence $\left\{x_{n}\right\}$ in the following manner $[11,12]$ :

$x_{1}=\beta_{1} f\left(x_{0}\right)+\left(1-\beta_{1}\right) x_{0}$

$x_{2}=\beta_{2} f\left(x_{1}\right)+\left(1-\beta_{2}\right) x_{1}$

.

$x_{n}=\beta_{n} f\left(x_{n}-1\right)+\left(1-\beta_{n}\right) x_{n}-1$

where $0<\beta_{n}<=1$ and $\left\{\beta_{n}\right\}$ is convergent away from 0 .

The sequence $\left\{\mathrm{x}_{\mathrm{n}}\right\}$ constructed this way is called a superior sequence of iterates, denoted by $S O\left(f, x_{0}, \beta_{n}\right)$ At $\beta_{n}=1, S O\left(f, x_{0}\right.$, $\beta_{n}$ ) reduces to $O\left(f, x_{0}\right)$.

\section{B. Definition}

(Superior Orbit) The sequences $x_{n}$ constructed above is called Mann sequence of iteration or superior sequences of iterates. We denote it by $\operatorname{SO}\left(x_{0}, s, t\right)[11,12]$.

This procedure was essentially given by Mann, was the first to study it for $\beta_{n}$ in 1955. 
Since the results obtained in fractal modelling via Mann iterates are the super set of their corresponding fractal models in the Picard orbit. Researchers have since developed superior fractal models for $\beta_{n}=\beta, n=1,2, \ldots$, for various values of $\beta$.

\section{Definition}

(Superior Julia sets) The set of complex points SK whose orbits are bounded under superior iteration of a function $Q$ is called the filled superior Julia set. A superior Julia set SJ of $Q$ is the boundary of the filled superior Julia set SK [8].

\section{Definition}

(Superior Mandelbrot sets) A Superior Mandelbrot set SM for a function of the form $Q_{c}(z)=z_{n}+c, n=1,2, \ldots$, is defined as the collection of $c \in C$ for which the superior orbit of the point 0 is bounded,

$\mathrm{SM}=\left\{c \in C:\left\{Q_{c}^{k}(0): k=0,1, \ldots \ldots \ldots\right\}\right.$ is bounded in $\left.\mathrm{SO}\right\}$ [9].

\section{E. Definition}

Suppose $x_{0}$ is a fixed point for $F$. Then $x_{0}$ is an attracting fixed point if $\left|F^{\prime}\left(x_{0}\right)\right|<1$. The point $x_{0}$ is a repelling fixed point if $\left|F^{\prime}\left(x_{0}\right)\right|>1$. Finally if $\left|F^{\prime}\left(x_{0}\right)\right|=1$, the fixed point is neutral [10].

\section{GENERATING THE FRACTAL}

The Mandelbrot set is the collection of $C$ points for which the orbit is bounded. The set of those points are known as prisoner set and remaining points comes under escape set. The escape criteria for the function $\sin \left(z^{n}\right)+c$ is given as follows:

\section{A. Escape Criteria for Quadratic Function:}

For $n=2$, the escape criteria is depends on a constant value or $(z>=1 / \omega)$.

\section{B. Escape Criteria for Cubic Polynomial:}

The escape criteria for the cubic polynomials by using Mann iteration for $\mathrm{n}=3$, the escape criteria is $(z>=((1+b) / 2$ $\left.\omega)^{1 / 2}\right)$.

\section{Escape Criteria for General Polynomial:}

The escape criteria for the general polynomial equations using Mann iteration procedure for $n$ is $z \geq\left(\frac{1}{\omega}\right)^{\frac{1}{n-1}}$.

Note that the initial value $z_{0}$ should be infinity, since infinity is the critical point of $z$ for $\sin \left(z^{n}\right)+c$. However instead of starting with $z_{0}=\infty$, it is simpler to start with $z_{1}=c$, which yields the same result. A critical point of $z \rightarrow f(z)+c$ is a point where $f^{\prime}(z)=0$. The point $\mathrm{z}$ in Mandelbrot set for sine function has an orbit that satisfies $\operatorname{imag}(z)>50$, then the orbit of $z$ escapes [2].

\section{GEOMETRY OF SUPERIOR TRANSCENDENTAL MANDELBROT SETS AND SUPERIOR TRANSCENDENTAL JULIA SETS:}

The fractal generated by this iteration process possesses symmetry about $x$ axis in case of all polynomials.

\section{A. Description of Superior Transcendental Mandelbrot Set:}

The fractal is symmetrical about $x$ axis for all values of $s$. Initially for a quadratic polynomial the value of $\mathrm{s}=1$ has been taken and got the fractal with two bulbs. As the value of $s$ has been changed from 1 to 0.3 , both bulbs merged together and resultant fractal is not very much sharp. There are very few tiny bulbs attached to primary bulbs for $s=0.5$. Subsequently we change the value of $s$ to 0.7 ; the primary bulb is showing too much tiny bulbs with attached decorations.

For a cubic polynomial, fractal shows its beautiful images. Starts with the value of $s=0.1$, and move towards 0.3 , we got an image of lord Vishnu according to Hindu mythology. With the $s=0.5$, there is an image of sparkling earthen lamp (diya) with its own image has been shown. There is a symmetrical image about $x$ axis for $s=0.7$.

For a biquadrate polynomial, the fractal is having three primary bulbs and large number of secondary bulbs attached to it. For $s=0.7$, shape of primary bulbs are approximately same as with the value of $s=1$ but the number and shapes of secondary bulbs reduced. In case of $s=0.3$ and 0.5 , the shapes of primary bulbs are same but the axis of secondary bulb varies based on above mentioned values of $s$.

\section{B. Description of Superior Transcendental Julia Set:}

Transcendental function $\sin (\mathrm{z})$ with superior Julia set, which follows the law of having $2 \mathrm{n}$ wings has analyzed, where $\mathrm{n}$ is the power of $\mathrm{z}$. The images for all polynomials possesses symmetry about both $\mathrm{x}$ and $\mathrm{y}$ axis. Here extremely beautiful images of superior Julia set have been generated for different power of polynomial.

An image of superior Julia set has a resemblance with hippocampus for a quadratic polynomial. In case of cubic polynomial, a star fish shape has formed with large central body having rotational and reflection symmetry along with axes symmetry. Finally a biquadrate polynomial is having same structure as cubic polynomial with eight wings.

\section{Generation of Superior Transcendental Mandelbrot Sets \\ For Quadratic function:}

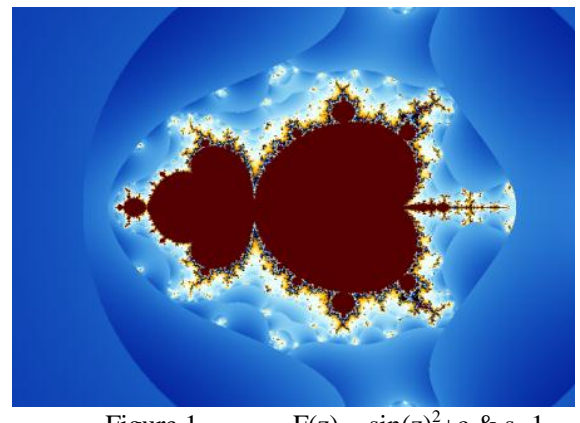

Figure 1. $\quad \mathrm{F}(\mathrm{z})=\sin (\mathrm{z})^{2}+\mathrm{c} \& \mathrm{~s}=1$ 


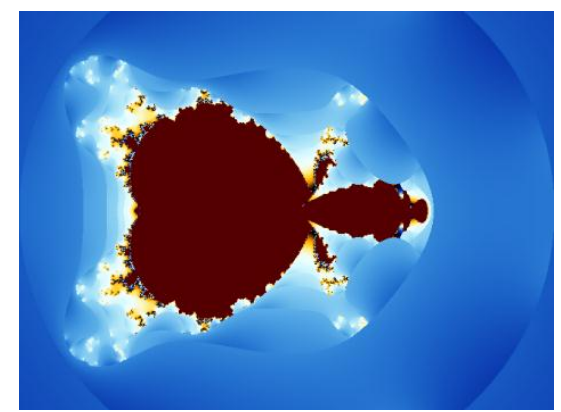

Figure 2.

$\mathrm{F}(\mathrm{z})=\sin (\mathrm{z})^{2}+\mathrm{c} \& \mathrm{~s}=0.3$

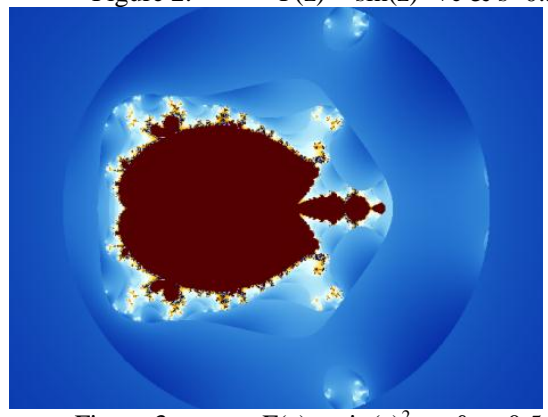

Figure 3.

$\mathrm{F}(\mathrm{z})=\sin (\mathrm{z})^{2}+\mathrm{c} \& \mathrm{~s}=0.5$

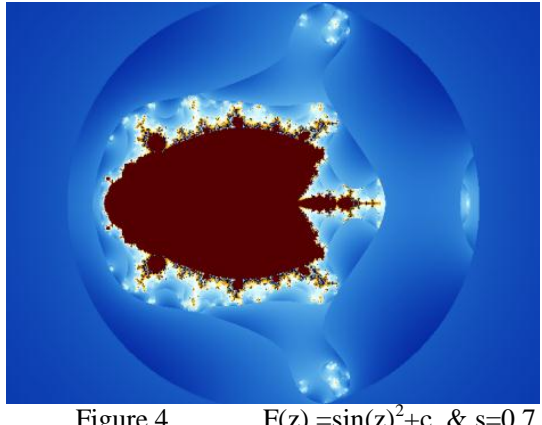

Figure 4.

$\mathrm{F}(\mathrm{z})=\sin (\mathrm{z})^{2}+\mathrm{c} \quad \& \mathrm{~s}=0.7$

For Cubic function:

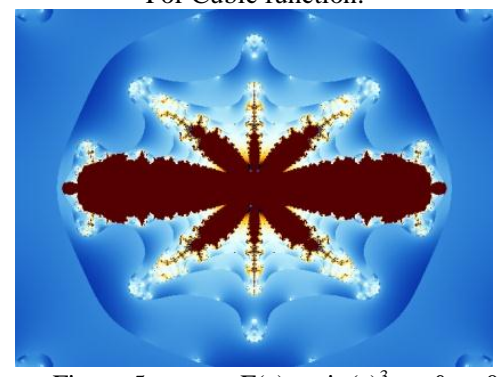

Figure 5 .

$\mathrm{F}(\mathrm{z})=\sin (\mathrm{z})^{3}+\mathrm{c} \& \mathrm{~s}=0.1$

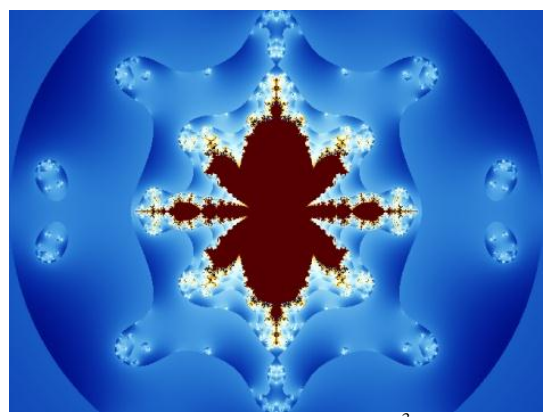

Figure 6.

$\mathrm{F}(\mathrm{z})=\sin (\mathrm{z})^{3}+\mathrm{c} \& \mathrm{~s}=0.3$

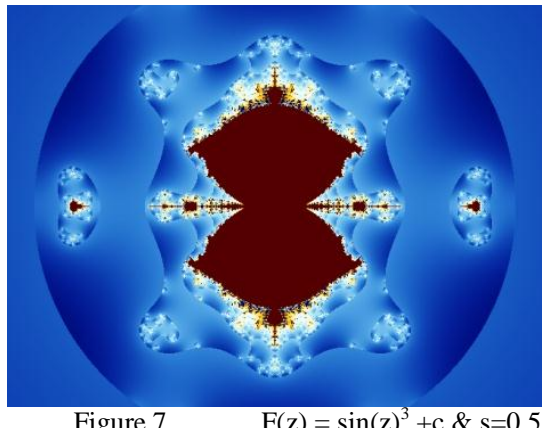

Figure 7.

$\mathrm{F}(\mathrm{z})=\sin (\mathrm{z})^{3}+\mathrm{c} \& \mathrm{~s}=0.5$

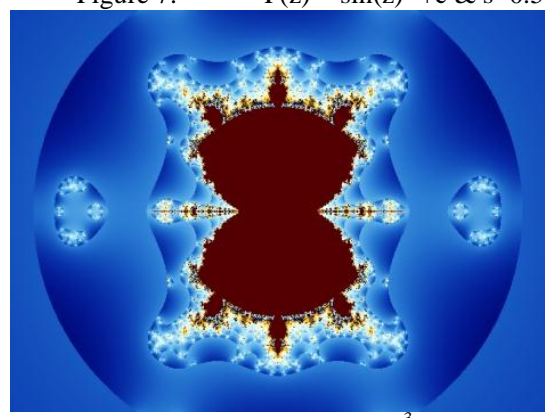

Figure 8.

$\mathrm{F}(\mathrm{z})=\sin (\mathrm{z})^{3}+\mathrm{c} \& \mathrm{~s}=0.7$

For Biquadratic function:

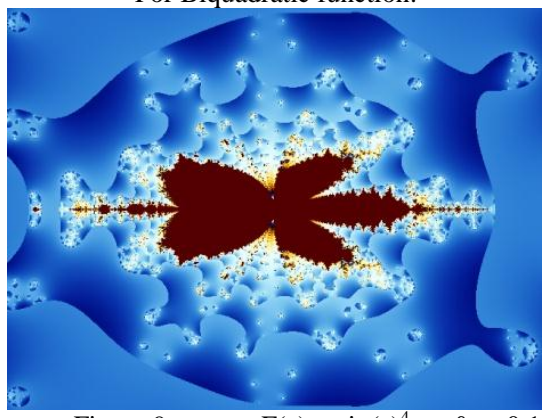

Figure 9.

$\mathrm{F}(\mathrm{z})=\sin (\mathrm{z})^{4}+\mathrm{c} \& \mathrm{~s}=0.1$

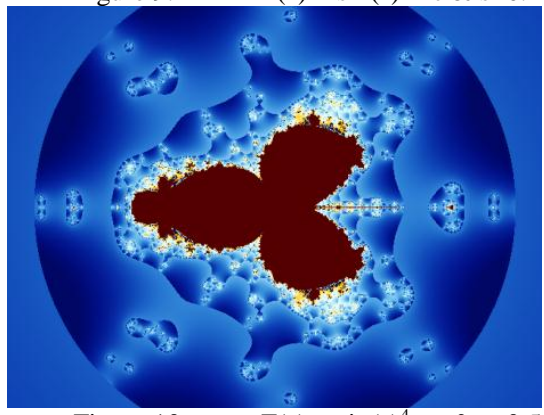

Figure 10

$\mathrm{F}(\mathrm{z})=\sin (\mathrm{z})^{4}+\mathrm{c} \& \mathrm{~s}=0.5$

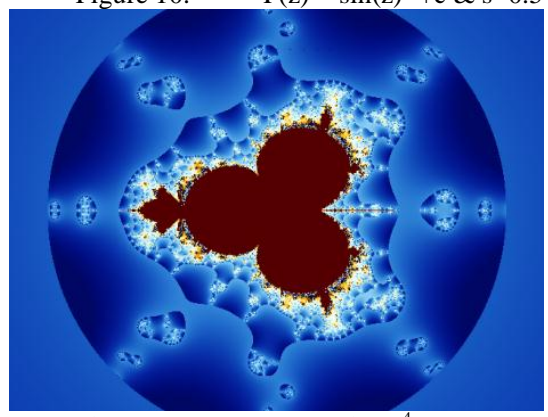

Figure 11

$\mathrm{F}(\mathrm{z})=\sin (\mathrm{z})^{4}+\mathrm{c} \& \mathrm{~s}=0.7$ 
D. Generation of Superior Transcendental Julia sets: For Quadratic Function:

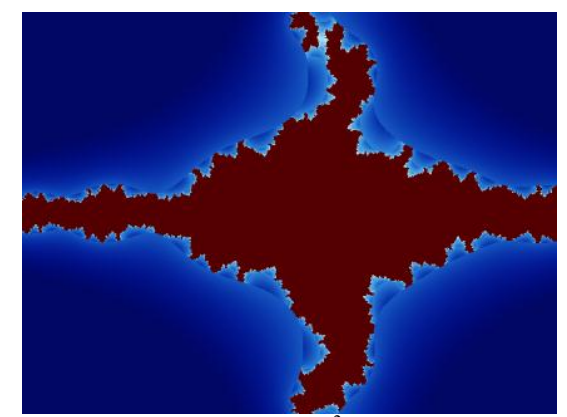

Figure 12. $\mathrm{F}(\mathrm{z})=\sin (\mathrm{z})^{2}+\mathrm{c}, \mathrm{s}=0.7$ and $\mathrm{C}=0.01902$, 0.17618

For Cubic Function:

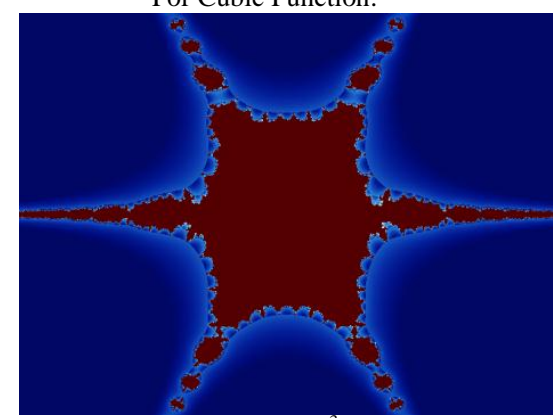

Figure 13. For $\mathrm{F}(\mathrm{z})=\sin (\mathrm{z})^{3}+\mathrm{c}, \mathrm{s}=0.7$ and $\mathrm{C}=0.77083$,$0.83 \mathrm{i}$

For Biquadratic function:

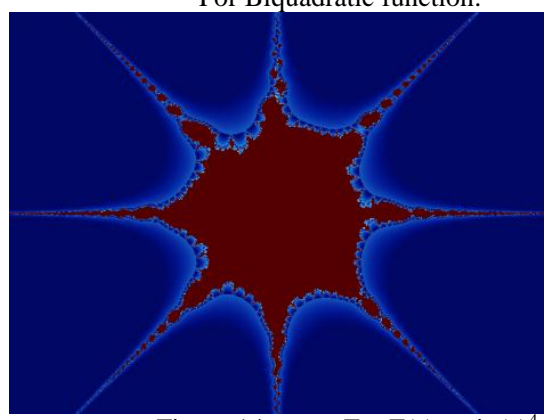

Figure 14. $\quad$ For $\mathrm{F}(\mathrm{z})=\sin (\mathrm{z})^{4}+\mathrm{c}$, $\mathrm{s}=0.7$ and $\mathrm{C}=-0.025,0.3125$

\section{E. Fixed Points}

Fixed points of Quadratic function:

TABLE I. Orbit of $\mathrm{F}(\mathrm{z})$ at $\mathrm{s}=0.7$,

\begin{tabular}{|l|l|l|l|}
\hline $\begin{array}{l}\text { Number of } \\
\text { Iteration i }\end{array}$ & $\mathbf{F}(\mathbf{z})$ & $\begin{array}{l}\text { Number of } \\
\text { Iteration i }\end{array}$ & $\mathbf{F}(\mathbf{z})$ \\
\hline 1 & 0.5396 & 7 & 0.5080 \\
\hline 2 & 0.4949 & 8 & 0.5080 \\
\hline 3 & 0.5130 & 9 & 0.5080 \\
\hline 4 & 0.5061 & 10 & 0.5080 \\
\hline 5 & 0.5087 & 11 & 0.5080 \\
\hline 6 & 0.5077 & 12 & 0.5080 \\
\hline
\end{tabular}

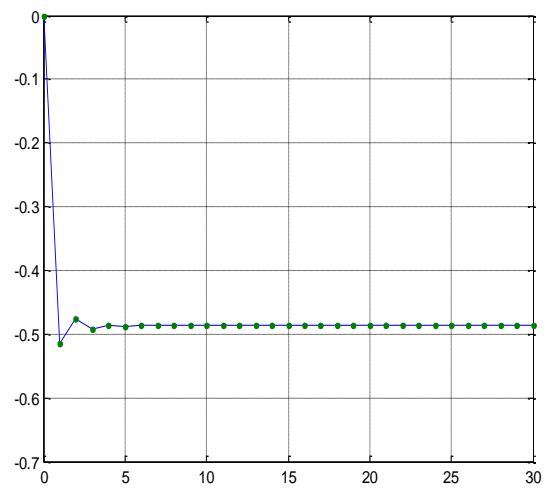

Figure 15. Orbit of $\mathrm{F}(\mathrm{z})$ at $\mathrm{s}=0.7$ for $(\mathrm{C}=-0.7708333333$ -

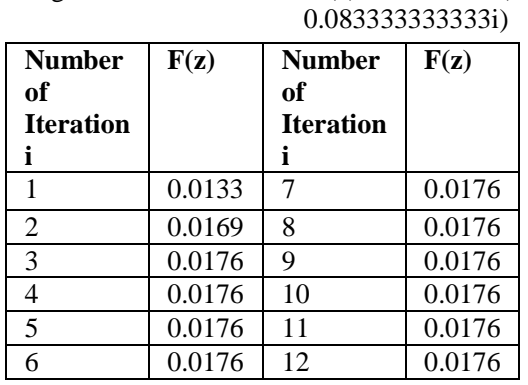

TABLE II. ORBIT OF F(z) AT S=0.7 FOR $(\mathrm{C}=0.01902+0.17618)$

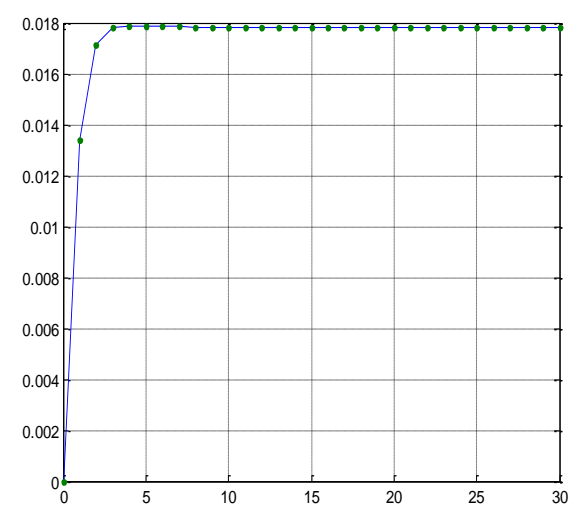

Figure 16. Orbit of $\mathrm{F}(\mathrm{z})$ at $\mathrm{s}=0.7$ for $(\mathrm{C}=0.01902,0.17618)$

Fixed points of Biquadratic function:

TABLE III. Orbit of $\mathrm{F}(\mathrm{z})$ at $\mathrm{s}=0.7$ for

\begin{tabular}{|l|l|l|l|}
\multicolumn{5}{|c}{$(\mathrm{C}=-0.025+0.3125)$} \\
\hline $\begin{array}{l}\text { Number of } \\
\text { Iteration } \mathbf{i}\end{array}$ & $\mathbf{F}(\mathbf{z})$ & $\begin{array}{l}\text { Number of } \\
\text { Iteration } \mathbf{i}\end{array}$ & $\mathbf{F}(\mathbf{z})$ \\
\hline 1 & 0.0175 & 7 & 0.0156 \\
\hline 2 & 0.0213 & 8 & 0.0155 \\
\hline 3 & 0.0197 & 9 & 0.0154 \\
\hline 4 & 0.0176 & 10 & 0.0154 \\
\hline 5 & 0.0164 & 11 & 0.0154 \\
\hline 6 & 0.0158 & 12 & 0.0154 \\
\hline
\end{tabular}




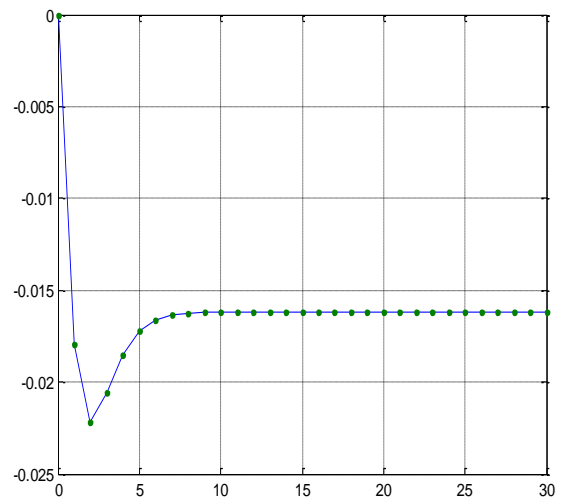

Figure 17. Orbit of $\mathrm{F}(\mathrm{z})$ at $\mathrm{s}=0.7$ for $(\mathrm{c}=-0.025+0.3125)$

\section{CONCLUSION}

In this paper, we have analyzed sine function in Mandelbrot equation with Mann iteration. Superior Julia set possess $2 \mathrm{n}$ wings with central black region. Our study shown the striking properties and escape criteria for transcendental function and generated the corresponding fractals using Mann iterates in which most of the images are having symmetry along $\mathrm{x}$ axis and $\mathrm{y}$ axis. The images revealed their own identity. As on a particular value of constant s, an image resembled to Lord Vishnu icon according to Hindu Mythology see Fig. [6]. Another image shows earthen lamp (diya) with its own reflection along with real axis see Fig. [7]. We obtained fixed point for quadratic function after 7 iterations, for cubic function after 3 iterations and for biquadratic function after 9 iterations.
The surrounding region of superior Mandelbrot set shown to be invariant cantor set in the form of curve or hair that tends to infinity under iteration in all figures.

\section{REFERENCES}

[1] A. Ereneko, "Iteration of entire function" Dynamical System \& Ergodic Theory, Banach Center publ. 23, polish Sc. Publ., Warsaw 1989, 339345.

[2] B. B. Mandelbrot, "The Fractal Geometry of Nature", W. H. Freeman, NewYork, 1983.

[3] Folkert Tangerman, R. L. Devaney, "Dynamics of Entire Function near the Essential Singularity", Ergodic Theory \& Dynamical System 64 (19860 489-503.

[4] H.O. Peitgen and P.H. Richter, The beauty of fractals, Springer Verlag, 1986.

[5] Kin-Keung Poon, "Fatuo-Julia Theory on Transcendental Semigroups", Bull Austrad. Math Soc. Vol. 58 (1998) [403-410].

[6] Kin-Keung Poon, "Dynamics on Transcendental Semigroups", (1991).

[7] M. Abbas, Sagar Hussain Khan, B.E. Rhoades, "Simpler is also better in approximating fixed points", applied Mathematics \& Computation 205 (2008), 428-431.

[8] Mamta Rani \& Vinod Kumar, "Superior Julia Set", J. Korea Soc. Math. Educ. Ser. D; Res. Math. Educ., 8(4), 2004, 261-277.

[9] Mamta Rani \& Vinod Kumar, "Superior Mandelbrot Set", J. Korea Soc. Math. Educ. Ser. D; Res. Math. Educ., 8(4), 2004, 279-291.

[10] R.L Devaney, "A First Course in Chaotic Dynmical System: Theory \& Experiment", Addision-Wesley, 1992. MR1202237.

[11] R. Rana, Y. S. Chauhan \& A. Negi, "Generation of New Fractals for Sin Function”, Int. J. Comp. Tech. Appl., vol2(6), 1747-1754 ISSN 22296093.

[12] R. Rana, Y. S. Chauhan \& A. Negi, "Generation of New Julia sets and Mandelbrot sets for Tangent Function", Computer Engineering \& Intelligent Systems, ISSN 2222-2863(online) vol2, No.8, 2011. 